\title{
HIERARCHICAL MRF MODEL FOR MODEL-BASED MULTI-OBJECT TRACKING
}

\author{
Yunqiang Chen and Thomas S. Huang \\ chenyq AT ifp.uiuc.edu, huang AT ifp.uiuc.edu \\ Beckman Institute, ECE , University of Illinois \\ Urbana, IL, USA 61801
}

\begin{abstract}
To track multiple objects, top-down (model-based methods) and bottom-up (multi-layer analysis) methods have been proposed separately. In this paper, a hierarchical MRF model is proposed to integrate these two trends into a MAP framework for tracking non-rigid objects such as human hands or faces. Parametric models of color, shape and frame difference for both foreground and background are given. Dynamic constraints are used to update the observation models and present an initial segmentation for the new frame. A novel hierarchical MRF model is proposed to efficiently refine the segmentation based on local smoothness constraints. The algorithm does not need to initialize and can detect new moving objects and track them. It can also handle the stopped objects because of the utilization of spatial-temporal constraints. Promising results are reported in this paper.
\end{abstract}

\section{INTRODUCTION}

Human motion analysis has become more and more important. Real-time applications such as video surveillance, human-computer intelligent interface and video conferencing all require the ability to track moving objects. An efficient multiple objects tracking algorithm in complex environments is a challenging task.

Both model-based methods and data-driver methods have been proposed. The model-based tracking methods is widely used to track human bodies, which are often represented as stick figures, 2D contours or volumetric blobs connected by joints $[1,2,3]$. Both dynamic and kinematic properties are incorporated to give more reasonable and accurate results. However, the models are always not so accurate. For background, no simple model is good enough. Background subtraction is hard to use in many environments.

To deal with complex background, unsupervised learning technique is used in [4] for color tracking. Mixture gaussian models for foreground and background are learned by discriminant EM algorithm for each new frame. But the ability to track multiple objects is not clear. To achieve better results, spatial-temporal constraints are necessary. In [1], they use some ad-hoc methods such as smoothing the class likelihood and using morphology to refine the support maps.

The data-driver methods, Multi-layer motion analysis, rely on MRF model to deal with spatial-temporal constraints. Various spatial constraints on layer motion and layer segmentation have been proposed [5, 6, 7]. But such kind of methods usually cost heavy computation and have to estimate the number of the layers, the motion parameters of each layer and the support maps from weak prior constraints. The heavy computation cost prevents this kind of methods to be used in real time. Works to integrate global prior model and local appearance model are done in [8], where Tao etc. propose dynamic layer representation for tracking rigid motion of vehicles.

In this paper, we integrate the model-based method and multi-layer motion estimation into a hierarchical MRF framework to track non-rigid motion of human hands and faces in an immersive desk environment. The rest of the paper is organized as follows. In Section 2, we define the parametric models of the observations and explain the hierarchical MRF structure. In Section 3, the tracking diagram based on dynamic constraints is given. We test our algorithm with real sequences and report promising results in Section 4. Concluding remarks are in Section 5.

\section{MATHEMATICAL FORMULATION}

Many real time algorithms use background subtraction to detect foreground. But background maintenance is difficult and is easy to fail. We use frame difference and color information to track hands and faces. In this section, we give the parametric models for foreground objects and background and explain how to integrate them with the temporal-spatial constraints into MAP framework.

\subsection{The Likelihood Function}

First we assume that each object (hands, faces) can be approximated by a coherent color blob [1]. The set of blob hypotheses is represented as a mixture of multivariate Gaussians. Each single Gaussian $\theta_{k}(t)$ encodes the coherent 
color value, the centroid and second moments of a blob. An additional layer $\theta_{0}(t)$ which has uniform distribution is defined for background. Suppose we know the parameters of each blob, tracking the objects is to classify every pixel in current frame to a blob.

The observation of each pixel includes the color $I(t, x, y)$, the position $(x, y)$ and the frame difference of each pixel. $\mathrm{YU}^{*} \mathrm{~V}^{*}$ color space [1] is used in our algorithm. For simplicity, we assume the distribution of color is Gaussian. The spatial proximity likelihood function for blob $k, P\left(x, y \mid \theta_{k}(t)\right)$, is also a Gaussian. Assuming the color distribution is independent of its position. The likelihood function for the objects can be defined as: (we define $s=(x, y)^{T}$ )

$$
\begin{aligned}
P\left(I, s \mid \theta_{k}(t)\right)= & \frac{e^{-\left(\frac{1}{2}\left(I-\mu_{c}\right)^{\prime} K_{c}^{-1}\left(I-\mu_{c}\right)\right)}}{\sqrt{(2 \pi)^{3} K_{c}}} \\
& \cdot \frac{e^{-\left(\frac{1}{2}\left(s-\mu_{s}\right)^{\prime} K_{s}^{-1}\left(s-\mu_{s}\right)\right)}}{\sqrt{(2 \pi)^{2} K_{s}}}
\end{aligned}
$$

To discriminate the moving foreground and static background (for moving background, motion compensation could be applied first), we employ 3 consecutive frames, $I_{t-1}, I_{t}$, $I_{t+1}$, to calculate both the forward difference map $\left(e^{f}=\right.$ $\left.I_{t+1}-I_{t}\right)$, and backward difference map $\left(e^{b}=I_{t}-I_{t-1}\right)$. Moving foreground pixels tent to have large $e^{b}$ and $e^{f}$, while background will have either small $e^{b}$ or small $e^{f}$. If we assume the independence among the frame difference, color distribution and position, we get the likelihood function for each class $P\left(a=\left(e, I_{x y}, x, y\right) \mid \lambda\right)$ : ( $\lambda_{0}$ is the label for unchanged pixels and $\lambda_{1}$ for changed pixels.)

Background pixels: (labeled as $\lambda_{B}$ )

$$
p\left(a \mid \lambda_{B}\right)=p\left(e^{f} \mid \lambda_{0}\right) p\left(e^{b} \mid \lambda_{0}\right) P\left(I, x, y \mid \theta_{0}(t)\right)
$$

Uncovered Background: (labeled as $\lambda_{U B}$ )

$$
p\left(a \mid \lambda_{U B}\right)=p\left(e^{f} \mid \lambda_{0}\right) p\left(e^{b} \mid \lambda_{1}\right) P\left(I, x, y \mid \theta_{0}(t)\right)
$$

Will-be-covered Background: (labeled as $\lambda_{C B}$ )

$$
p\left(a \mid \lambda_{C B}\right)=p\left(e^{f} \mid \lambda_{1}\right) p\left(e^{b} \mid \lambda_{0}\right) P\left(I, x, y \mid \theta_{0}(t)\right)
$$

Foreground kth blob: (labeled as $\lambda_{F k}$ )

$$
p\left(a \mid \lambda_{F k}\right)=p\left(e^{f} \mid \lambda_{1}\right) p\left(e^{b} \mid \lambda_{1}\right) P\left(I, x, y \mid \theta_{k}(t)\right)
$$

In our experiment, we use a binary model for the the frame difference of changed and unchanged pixels:

$$
\begin{aligned}
& p\left(e \mid \lambda_{0}\right)= \begin{cases}1 / 6 & \text { if } e>t h \\
5 / 6 & \text { if } e<t h\end{cases} \\
& p\left(e \mid \lambda_{1}\right)= \begin{cases}5 / 6 & \text { if } e>t h \\
1 / 6 & \text { if } e<t h\end{cases}
\end{aligned}
$$

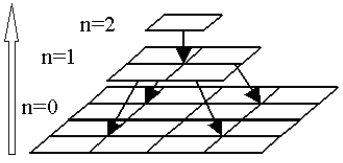

(a)

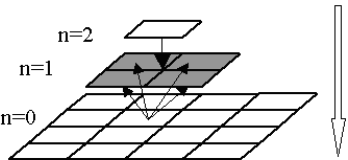

(b)
Fig. 1. Structure of Hierarchical MRF Model

We set the parameters by hand and it gives us very good results. Further detailed model or some adaptive methods can be used to get more accurate models.

Given all the likelihood function, the best class for each pixel may be computed using the standard MAP probability decision rule:

$$
\widehat{\lambda}=\arg \max _{\lambda \in \Lambda} p(\lambda \mid a)=\arg \max _{\lambda \in \Lambda} p(a \mid \lambda) p(\lambda)
$$

Some model-based tracking algorithms (i.e. [1, 2, 3]) assume $p(\lambda)$ of each pixel is uniform and independent from its neighbors and try to classify each pixel to different blobs considering only the $p(a \mid \lambda)$. Unfortunately, this assumption is not right. The $p(\lambda)$ of each pixel correlates with the labels of its spatial-temporal neighbors, e.g. that the support map of each object tends to be smooth and the label of one pixel tends to remain the same if its color does not change from previous frame. Such spatial-temporal constraints can be represented as a MRF model.

\subsection{Hierarchical MRF Model}

We design a new efficient hierarchical MRF model to classify all the pixels based on the prior model and the spatial constraints. Traditional hierarchical MRF models, e.g., $[10,11]$, assume no prior knowledge at first. So they try to derive it from coarsest level in the pyramid and then use it as initial condition in the finer level. They still need many iterations to refine the initial condition at each finer level.

In tracking systems, the parameters for each object can be predicted by dynamic properties. We apply the predicted prior models to classify all the pixels in the new frame and assume the predicted prior models are good enough to give "confident" (e.g. $\frac{p\left(a \mid \lambda_{i}\right)}{\sum_{k} p\left(a \mid \lambda_{k}\right)}>t h$ ) labels to some pixels in the new frame $I_{t}$, so that the pixels in the new frame can be divided into labeled pixels and unlabeled pixels.

The pyramid is shown in Figure 1 (a). Each node in it has 4 children in the lower level. Using the labeled pixels, we start building the pyramid from bottom level. The parent node in the upper level takes the label that most of its children have. If most of its children are unlabeled, the parent is also unlabeled. The observation $\left(a_{s}\right)$ is the average of those children's observations. This process continue from the finest level to the coarsest level (4 levels in our experiments). 
Suppose all the nodes in the top level are labeled (if not, we can do it very efficiently with a traditional MRF), we try to classify only the unlabeled nodes from top to the bottom. To do this efficiently, a new neighborhood is defined in the pyramid. Each node in the pyramid has 4 neighbors in the higher level as shown in Figure 1 (b). A common smoothness constraint energy term like (1) can still be used with this new neighborhood definition.

$$
\begin{gathered}
E\left(\lambda_{s}\right)=\sum_{s_{1} \in N} \alpha_{d}\left\|a_{s_{1}}-a_{s}\right\|^{2} \delta\left(\lambda_{s_{1}}-\lambda_{s}\right) \\
+\alpha_{s}\left(1-\delta\left(\lambda_{s_{1}}-\lambda_{s}\right)\right)
\end{gathered}
$$

Where $N$ is the set of all the new neighbors. $s$ is the unlabeled node and $s_{1}$ is one of its neighbors. $\alpha_{d}$ and $\alpha_{s}$ are two coefficients. The delta function in the first term suspends the smoothness constraint across region boundaries. The second term favors compact regions with short boundaries. The unlabeled node is classified to the $\hat{\lambda}$ that give the minimum energy of $\left(-\log p\left(a_{s} \mid \lambda_{s}\right)+E\left(\lambda_{s}\right)\right)$. After all the pixels in the finest level are classified, we can reestimate the global prior models for each object and move on to next frame.

In this new model, a pyramid is build upon the "confidently" labeled pixels. A new neighborhood is defined in the pyramid structure to increase the size of the neighborhood. And hence a non-recursive method can be derived. Automatic adaptation to the color changes is also achieved because the pyramid is also build on the observation of current frame.

\section{TRACKING ALGORITHM}

Besides the spatial constraints, global temporal coherence (dynamic constrain) is also used in global blob model update. Because the estimation of the global parameters may have noise, we use Kalman Filter [12] to smooth the new position and orientation of each blob:

$$
\widehat{X}_{[n \mid n]}=\widehat{X}_{[n \mid n-1]}+\widehat{G}_{[n]}\left(\widehat{Y}_{[n]}-\widehat{X}_{[n \mid n-1]}\right)
$$

where the estimated state vector includes the blob's position and velocity, the observations are the centroid of the blobs in current image, and the Kalman gain matrix assume simple Newtonian dynamics.

The tracking algorithm works in following way. For every image $I_{t}$, we compute the forward and backward error. Then use hierarchical MRF model to classify each pixel. But because of the introduction of blob model, we can fully utilize the dynamic constrain of the object motion. The global model also gives us a much better initial classification result and hence the new pyramid structure can be build.

Initialization for the new intruding objects is also addressed in this paper. After classifying the pixels in the image with detected blobs, we analysis all the moving pixels

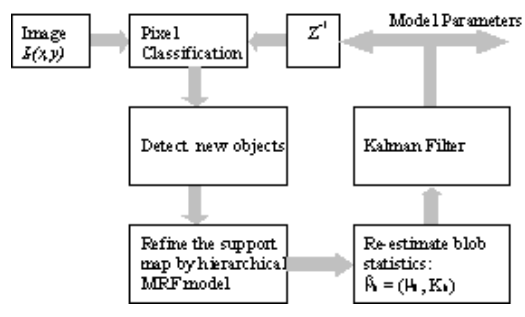

Fig. 2. Diagram of tracking algorithm

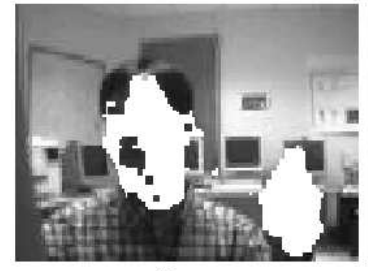

(a)

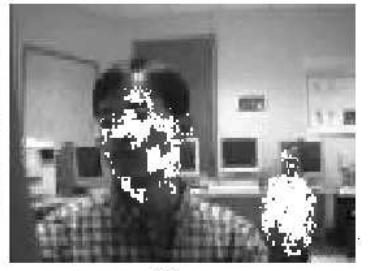

(b)
Fig. 3. The comparison of the support map (a) Using MAP framework (b) Without MAP framework

that can not be classified into any existing blobs and try to interpret them with a new blob if their color is close to skin color. The simple block diagram in Figure 2 summarizes the tracking algorithm described in this section.

\section{EXPERIMENTS}

Experiments on real sequences have been shown in Figure 4 . It shows very promising results even when multiple persons are in the scene. In the experiment, the different objects are indicated by different ellipses.

In the first frame, there are only two objects (one face and one hand) tracked. In the next frame, the intruder's arm is detected and tracked with a new blob. The intruder's face is not tracked because it is smaller than the threshold of the size we set for the object to be tracked. When his arm is occluded in the fourth image, the algorithm stops tracking it. During this whole sequence, the waving hand and the rather static face of the user are well tracked, even when the intruder's arm is very close to the user's face. Without the spatial-temporal constraints, it would be very difficult to handle the occlusion of the intruder's arm.

The waving hand is rather easy to track because it kept moving around. But the face is difficult to track in this sequence. Because the lighting is not very well on the face, the color does not looks like face color. Also, the frame difference will not help because the face did not move much. Without local smooth constraints, it would be very difficult to get accurate support map of it. The comparison was given in Figure 3. 


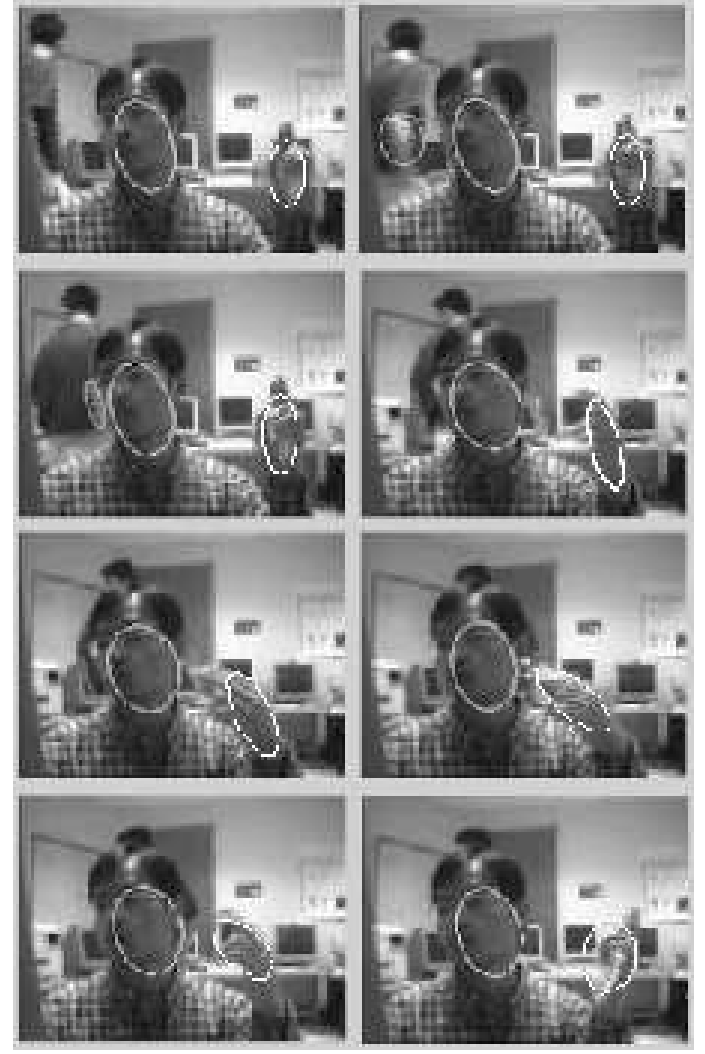

Fig. 4. Results of Tracking

\section{CONCLUSION}

In this paper, we integrate both the model-based method and a novel hierarchical MRF model to track multiple objects. It enables us to utilize both the object-level prior knowledge (such as the shape prior of the objects or dynamic properties of the motion) and pixel-level constraints (such as local spatial-temporal constraints or color distribution and noise models) during the tracking.

Not like the multi-layer motion estimation, we have the shape prior of the objects and know the dynamic properties of the motion to predict where the objects will be in the next frame. New pyramid model is developed to utilize these priors. The incorporation of spatial constraints gives us more accurate support map and hence more accurate estimation of the objects' position and properties. The algorithm is quite fast and has great potential for real time tracking.

\section{ACKNOWLEDGEMENTS}

This work was supported in part by National Science Foundation Grant CDA 96-24396, IIS-00-85980 and EIA 9975019.

\section{REFERENCES}

[1] C. Wren, A. Azarbayejani, T. Darrell, and A. Pentland, "Pfinder: Real-time tracking of the human body," IEEE Trans. on Pattern Analysis \& Machine Intell., vol. 19, no. 7, pp. 780-785, 1997.

[2] C. Bregler, "Learning and recognizing human dynamics in video sequences," in Proc. IEEE Int'l Conf. on Comput. Vis. and Patt. Recog., 1997, pp. 568-574.

[3] N. Jojic, M. Turk, and T. S. Huang, "Tracking articulated objects in dense disparity maps," in Proc. IEEE Int'l Conf. on Computer Vision, 1999, pp. 123-130.

[4] Y. Wu and T. S. Huang, "Color tracking by transductive learning," in Proc. IEEE Int'l Conf. on Comput. Vis. and Patt. Recog., 2000, pp. I:133-138.

[5] C. Stiller and J. Konrad, "Estimating motion in image sequences, a tutorial on modeling and computation of 2D motion," IEEE Signal Processing Magazine, vol. 16, no. 4, pp. 70-91, 1999.

[6] N. Vasconcelos, "Empirical Bayesian EM-based motion segmentation," in Proc. IEEE Int'l Conf. on Comput. Vis. and Patt. Recog., 1997, pp. 527-532.

[7] P. H. S. Torr, R. Szeliski, and P. Anandan, "An integrated Bayesian approach to layer extraction from image sequences," in Proc. IEEE Int'l Conf. on Comput. Vis. and Patt. Recog., 1999, pp. 983-990.

[8] H. Tao, H. S. Sawhney, and R. Kumar, "Dynamic layer representation and its applications to tracking," in Proc. IEEE Int'l Conf. on Comput. Vis. and Patt. Recog., June 2000, pp. 134-141.

[9] P. Giaccone, D. Amanatidis, and G.A. Jones, "Segmenting image sequences by embedding motion and colour cues within a contextual relaxation scheme," in IEE Colloquium on Motion Analysis and Tracking, London, UK, 1999, pp. 18/1-6.

[10] M. Mignotte, C. Collet, P. Perez, and P. Bouthemy, "Unsupervised hierarchical Markovian segmentation of sonar images," in Proc. IEEE Int'l Conf. Acoustic Speech Signal Processing, 1997, vol. 4, pp. 27812785.

[11] C. A. Bouman and M. Shapiro, "A multiscale random field model for Bayesian image segmentation," IEEE Trans. Image Processing, vol. 3, no. 2, pp. 162-177, 1994.

[12] H. V. Poor, An Introduction to Signal Detection and Estimation, New York, NY: Springer-Verlag, 1994. 